Artur Dias-Lima 1

Eloy Castellón Bermúdez 2 Jansen Fernandes de Medeiros 2 Italo Sherlock 1

\section{Estratificação vertical da fauna de flebótomos (Diptera, Psychodidae) numa floresta primária de terra firme da Amazônia Central, Estado do Amazonas, Brasil}

\author{
Vertical stratification of phlebotomine \\ sandfly fauna (Diptera, Psychodidae) \\ in a primary non-flooded forest of the \\ Central Amazon, Amazonas State, Brazil
}

\footnotetext{
1 Laboratório de Parasitologia/Entomologia Centro de Pesquisas Gonçalo Moniz, Fundação Oswaldo Cruz.

Rua Waldemar Fal cão 121, Salvador, BA 40295-001, Brasil. alima@cpqgm.fiocruz.br 2 Coordenação de Pesquisas em Ciências da Saúde, Instituto Nacional de Pesquisas da Amazônia. Av. André Araújo 2936, Manaus, AM 69011-970, Brasil.
}

\begin{abstract}
Studies on the vertical stratification of phlebotomine sandfly fauna were conducted in a non-flooded primary forest at a Tropical Forest Experimental Station of the National Institute of Amazonian Research from October 1998 to March 1999. CDC light traps were placed at one, 10, and 20 meters above ground. A total of 2,859 sandflies were captured, bel onging to the Lutzomyia (99.93\%) and Brumtomyia (0.07\%) genera, represented by 38 species. In the Lutzomyia gender, the most frequent sub-gender was Nyssomyia (43.4\%), followed by Psychodopygus (22.8\%). Lutzomyia umbratilis, L. anduzei, L. rorotaensis, L. trichopyga, and L. olmeca nociva predominated at one meter above ground, while L. davisi, L. infraspinosa, L. umbratilis, L. trichopyga, and L. anduzei predominated at 10 meters. L. anduzei, L. tuberculata, L. dendrophyla, and L. dreisbachi were the most abundant species at 20 meters. L. umbratilis, which appeared at all three levels of vertical stratification, has great epidemic significance as a vector of Leishmania (Viannia) guyanensis.
\end{abstract}

Key words Psychodidae; DiseaseVectors; Tropical Ecosystem; Amazonian Ecosystem

Resumo Estudos sobre a estratificação vertical da fauna de flebótomos, foram realizados numa floresta primária de terra firme, da Estação Experimental de Silvi cultura Tropical - Instituto Nacional de Pesquisas da Amazônia, durante os meses de outubro de 1998 a março de 1999. Utilizando-se armadilhas luminosas CDC, colocadas a 1, 10 e 20 metros de altura do solo, foram coletados 2.859 flebótomos, pertencentes a dois gêneros, Lutzomyia (99,93 \%) e Brumtomyia (0,07\%), abrangendo 38 espécies. Dentro do gênero Lutzomyia, os sub-gêneros mais representados foram Nyssomyia, com $43,4 \%$, ePsychodopygus com $22,8 \%$. As espécies Lutzomyia umbratilis, L. anduzei, L. rorotaensis, L. trichopyga e L. olmeca nociva, foram dominantes a $1 \mathrm{~m}$ de altura, enquanto que L. davisi, L. infraspinosa, L. umbratilis, L. trichopyga e L. anduzei, foram dominantes a $10 \mathrm{~m}$. As espécies L. anduzei, L. tuberculata, L. dendrophyla e L. dreisbachi foram mais abundantes a 20m. L. umbratilis, presente nos três níveis de estratificação vertical, é dotada de al ta importância epi demiológi ca como vetora da Leishmania (Viannia) guyanensis.

Palavras-chave Psychodidae; Vetores de Doença; Ecossi stema Tropical; Ecossistema Amazônico 


\section{Introdução}

Um dos critérios propostos por Chaniotis et al. (1971a), para incriminar espécies de flebótomos como potenciais vetoras de leishmanioses para o homem, além da densidade populacional correlacionada com o ritmo circadiano e sazonalidade, é a atividade de picadas ao nível do solo. Estudos que venham reconhecer a distribuição de espécies vetoras em vários níveis de estratificação vertical, são parâmetros que servem para determinar o grau de interação de um flebótomo com seu hospedeiro.

Na floresta tropical, o solo e a copa das árvores podem ser vistos como diferentes habitats, com componentes físicos e biológicos distintos. A copa das árvores, como um sítio primário de florescência e frutificação, atrai e abriga muitos vertebrados, tais como primatas e preguiças. As condições climáticas na copa das árvores são diferentes das do nível do solo, ou seja, há diferenças nos microclimas (temperatura, umidade relativa, intensidade de luz, movimento de ar, níveis de $\mathrm{CO}_{2}$, etc.). A distribuição dos flebótomos em vários níveis de estratificação vertical, é provavelmente, uma resposta a uma ou mais dessas diferenças físicas e biológicas (Chaniotis et al., 1971a). Um exemplo típico da influência de um desses fatores é descrito por Lainson (1983), no ritmo circadiano de Lutzomyia umbratilis Ward \& Fraiha, 1977. Nas primeiras horas da manhã, o aquecimento da copa das árvores pressiona os flebótomos a descerem ao nível do solo para protegerem-se da dessecação. Ao entardecer, o resfriamento contínuo favorece o retorno dos flebótomos às copas das árvores, em busca das suas fontes de alimento, principalmente preguiças e tamanduás.

A composição vegetal das florestas tropicais influencia na distribuição das espécies de flebótomos, seja pela produção de efeitos distintos no solo (Rutledge \& Ellenwood, 1975) ou pelas características peculiares como ocos e cascas dos troncos das árvores (Cabanillas \& Castellón, 1999; Geoffroy et al., 1986).

Flebótomos que vivem ao nível do solo, podem vir a atuar como vetores de leishmanias para os mamíferos, incluindo o homem, porque à noite têm oportunidade de se alimentar em reservatórios de leishmanioses. É o caso da espécie Lutzomyia flaviscutellata Mangabeira, 1942, um flebótomo de vôo baixo, cujo principal hospedeiro éo roedor Proechimys guyanensis, além de marsupiais, outros roedores e às vezes raposas (Lainson, 1983). Segundo Ready et al., (1986) flebótomos que habitam ao nível do solo, também poderiam ser vetores ocasio- nais de leishmanias aos mamíferos, incluindose o homem, porque à noite eles têm a oportunidade de se alimentar em tamanduás, que viajam longas distâncias ao nível do solo, ou também em preguiças, que periodicamente descem para defecar.

A estratificação vertical dos flebótomos no Continente Americano foi estudada por Disney (1966), Williams (1970) e Chaniotis et al. (1971a, 1971b). Na Amazônia brasileira, tem sido estudada por diversos autores, entre eles, Arias \& Freitas (1982), Barrett (1993), Biancardi (1981), Castellón et al. (1989, 1991, 1994), Genaro et al. (1986), Shaw et al. (1972) e Silva (1993). A importância do estudo da estratificação está relacionada, principalmente, com a observação do grau de exposição humana às espécies silvestres de flebótomos vetoras de leishmanioses.

\section{Métodos}

As coletas dos flebótomos foram realizadas na Estação Experimental de Silvicultura Tropical, Instituto Nacional de Pesquisas da Amazônia (INPA), localizada nas coordenadas geográficas 20 47' 05" S e 600 11' 51" W, a 45km ao norte da cidade de Manaus, Estado do Amazonas (Figura 1). Uma descrição geomorfoclimática, florística e faunística (vertebrados) mais detal hada desta área pode ser encontrada em Jardim \& Hosakawa (1987) e Yabe et al. (1998).

Em uma área de floresta primária de terra firme (Figura 2), de aproximadamente 1ha, foi realizada a coleta dos flebótomos, utilizandose 18 armadilhas luminosas CDC “miniatura” (Hausher's MachineWorks, New Jersey, Estados Unidos), sendo colocadas seis armadilhas a 1, 10 e $20 \mathrm{~m}$ de altura do solo, no período de outubro de 1998 a março de 1999. As armadilhas foram colocadas durante quatro noites consecutivas por mês, no crepúsculo, entre 17:00 e 6:00 do dia seguinte, durante a fase de lua nova (Aguiar et al., 1985; Sherlock et al., 1996).

Inicialmente, foi demarcada no platô uma trilha principal de $100 \mathrm{~m}$ de comprimento. No início e no final da trilha principal foram delineados dois transectos paralelos, no sentido Sudeste-Noroeste, com comprimento de 50m para direita e para esquerda (trilhas A, B, C e D) (Figura 3). No final dessas trilhas, ou seja, nos transectos de $50 \mathrm{~m}$ de ambos os lados e na triIha principal, foram colocadas armadilhas a 1, 10 e $20 \mathrm{~m}$ de altura. Foram ao todo, seis pontos de coleta com armadilhas luminosas.

Para observar se existiam diferenças na estratificação vertical entre 1 e 10m de altura do solo, foi utilizado o teste estatístico Mann-Whit- 
ney, enquanto que, para comparar a estratificação a 1, 10 e 20m, foi utilizado o Kruskal Wallis. Os testes estatísticos só foram aplicados para as espécies mais abundantes.

\section{Resultados}

Foram coletados 2.859 exemplares de flebótomos, abrangendo 38 espécies e, dentre estas, 29 (74,3\%), têm hábito de picar o homem, principalmente as dos sub-gêneros Nyssomyia e Psychodopygus (Young \& Arias, 1992) (Tabela 1).

Um exemplar macho da espécie L. cayennensis Floch \& Abonnenc, 1941, foi a única que ocorreu exclusivamente a $1 \mathrm{~m}$ de altura do solo. Por outro lado, cinco espécies foram capturadas somente a 10m: 1 macho e 1 fêmea de Brumtomyia pintoi Costa Lima, 1932; 2 machos de L. bispinosa Fairchild \& Hertig, 1951; 1 macho de L. inflata Floch \& Abonnenc, 1944 e 5 fêmeas de L. inpai Young \& Arias, 1977.

Cinco espécies foram dominantes a $1 \mathrm{~m}$ de altura, que representaram aproximadamente $57,6 \%$ do total dos flebótomos coletados: L. umbratilis (18,6\%), L. rorotaensis Floch \& Abonnenc, 1944 (15,9\%), L. ol meca nociva Young \& Arias, 1982 (8,7\%), L. trichopyga Floch \& Abonnenc, 1945 (7,4\%) e L. anduzei Rozeboom, 1942 (7\%). Outras espécies foram dominantes a 10m de altura, L. davisi Root, 1934 (19,3\%), L. ayrozai Barretto \& Coutinho, 1942 (7,5\%), L. amazonensis Root, 1934 (6,9\%) e L. geniculata Man-

Figura 1

Mapa da Amazônia Central Brasileira, mostrando a localização da Estação Experimental de Silvicultura Tropical do Instituto Nacional de Pesquisas da Amazônia.

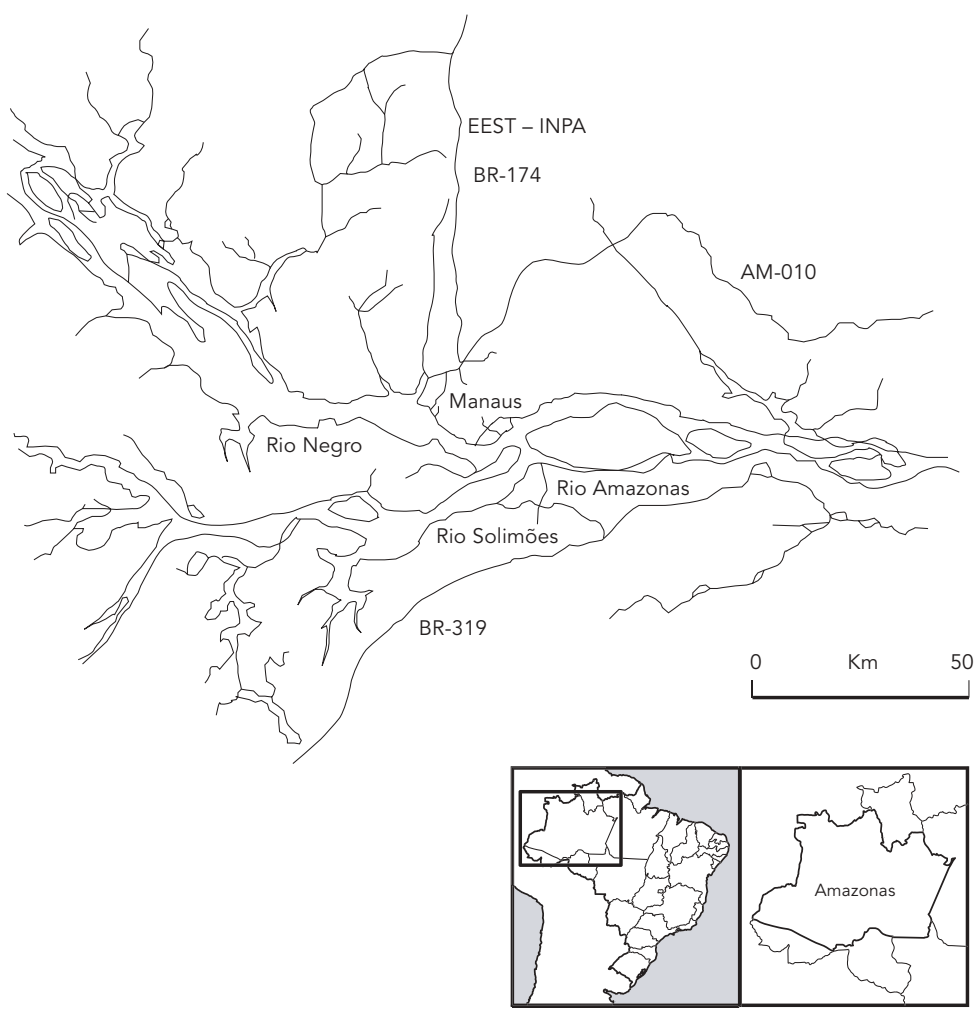

Figura 2

Corte transversal, mostrando as características do relevo da área de estudo.

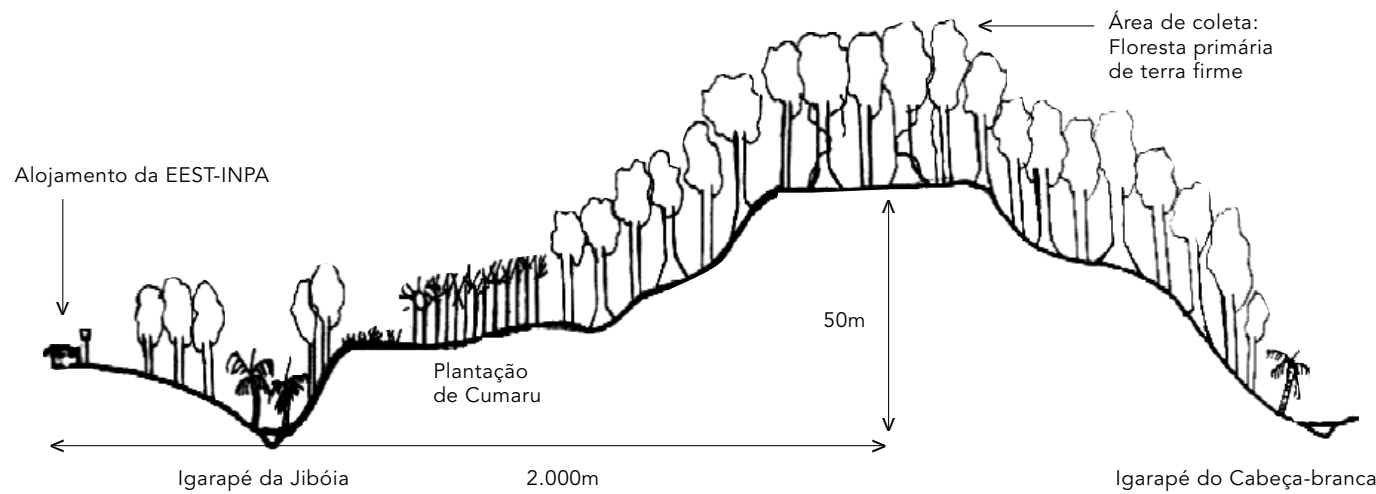


Figura 3

Desenho experimental do local de coleta com armadilhas luminosas colocadas a 1, 10 e 20 metros de altura, demonstrando a trilha principal e as trilhas secundárias $A, B, C$ e $D$ (transectos) com $50 \mathrm{~m}$.

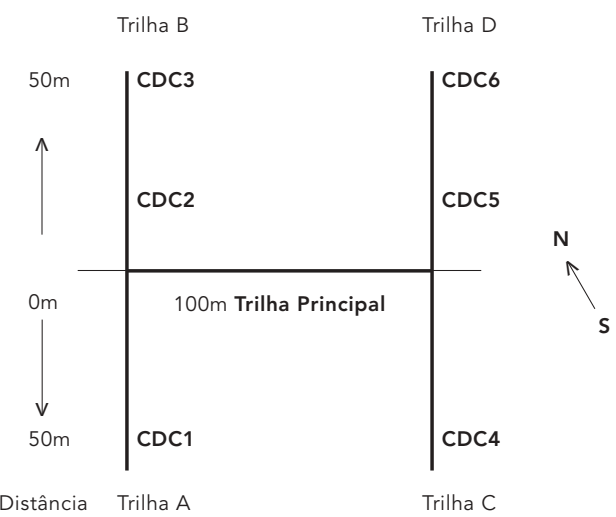

gabeira, 1941 (6,6\%), além das espécies que também foram dominantes a $1 \mathrm{~m}$ de altura, $\mathrm{L}$. umbratilis (15,8\%), L. anduzei $(6,6 \%)$ e L. trichopyga (5,2\%).

Quando foram comparados os níveis de estratificação vertical entre 1 e 10m de altura, as espécies L. infraspinosa Mangabeira, 1941, L. rorotaensis Floch \& Abonnenc, 1944, L. olmeca nociva Young \& Arias, 1982, L. nematoducta Young \& Arias, 1984, L. monstruosa Floch \& Abonnenc, 1944, L. sericea Floch \& Abonnenc, 1944 e L. flaviscutel lata, foram coletadas em maior número a $1 \mathrm{~m}$, demonstrando uma diferença significativa ( $p<0,001$ ) em relação ao número de indivíduos coletados a 10m de altura.

As espécies L. paraensis Costa Lima, 1941, L. dreisbachi Causey \& Damasceno, 1945, L. davisi , L. amazonensis, L. ayrozai e L. squamiventris squami ventris Lutz \& Neiva, 1912, foram mais abundantes a $10 \mathrm{~m}$ de altura, demonstrando uma diferença significativa $(p<0.001)$ em relação ao número de indivíduos coletados a $1 m$ de altura.

As espécies L. umbratilis, L. geniculata, L. furcata Mangabeira, 1941, L. dendrophyla Mangabeira, 1942, L. choti Floch \& Abonnenc, 1941, L. anduzei, L. aragaoi Costa Lima, 1932 e L. trichopyga, foram coletadas em números relativamente próximos nos diferentes níveis, não apresentando diferença significativa $(p<0.05)$, quando comparados o número de indivíduos coletados a 1 e $10 \mathrm{~m}$ de altura.
Comparando o total de espécimes coletados a 1, 10 e 20m de altura, observou-se uma diferença estatística significativa $(p=0.004)$ da altura de $20 \mathrm{~m}$, em relação ao número de indivíduos capturados a 1 e 10m de altura.

As espécies L. tuberculata, L. dendrophyla e L. anduzei, foram coletadas em maior número a $20 \mathrm{~m}$ apresentando uma diferença significativa ( $p<0,001)$, em relação ao número de indivíduos a 1 e $10 \mathrm{~m}$ de altura.

O sub-gênero mais representativo a $1 \mathrm{~m}$ de altura foi Nyssomyia (36,8\%), seguido de Psychodopygus (9,4\%) e Trichopygomyia (7,9\%). A 10m de altura, o sub-gênero Psychodopygus apareceu com maior freqüência $(47,6 \%)$, em relação aos outros sub-gêneros ou grupos, com Nyssomyia aparecendo em segundo $(24,2 \%)$ e Trichopygomyia em terceiro (5,8\%). O sub-gênero Nyssomyia teve presença marcante $(69,2 \%)$ a 20 metros, seguido de Viannamyia e Psychodopygus, com 8,8 e $8,3 \%$, respectivamente.

As espécies do sub-gênero Nyssomyia, L. umbratilis e L. anduzei, foram mais coletadas a $20 \mathrm{~m}$ na altura, enquanto que L. flaviscutellata e L. olmeca nociva a $1 m$ (Figura 4).

Todas as espécies do sub-gênero Psychodopygus foram coletadas com maior freqüência a $10 \mathrm{~m}$ de altura, onde L. davisi, L. ayrozai, L. geniculata $(p<0,05)$ e L. amazonensis ( $p<$ 0,001 ), apresentaram diferenças significativas, em relação a $1 \mathrm{~m}$. As espécies L. paraensi se L. squami ventris squamiventris não demonstraram diferenças estatísticas significativas quando comparados os três níveis de estratificação (Figura 5).

As espécies agrupadas em outros sub-gêneros, que tiveram uma atividade maior a $1 \mathrm{~m}$ de altura, foram L. monstruosa ( $p<0,01)$, L. trichopyga, L. nematoducta $(p<0,05)$ L. infraspinosa e L. rorotaensis ( $p<0,001)$ (Figura 6). As espécies L. tuberculata, $(p<0,01)$ e L. dendrophyla $(p<0,05)$ apresentaram diferença significativa, dando preferência a altura de 20m. Já para as espécies L. furcata e L. gomezi Nitzulescu, 1931, não foram registradas diferenças nos diversos níveis de estratificação vertical.

\section{Discussão}

De acordo com Arias \& Freitas (1982), num trabalho realizado na Reserva Ducke, INPA, na Amazônia Central, Castellón et al. (1989) em Roraima, e com os resultados deste trabalho, as espécies L. ayrozai, L. davisi, L. paraensis, L. dendrophyla, L. dreisbachi, L. shannoni, L. trichopyga, L. tuberculata eL. amazonensis, foram coletadas em maiores alturas (10 e 15m), sendo 


\section{Tabela 1}

Freqüência das espécies de flebótomos, em diferentes alturas, coletadas com armadilhas luminosas.

Estação Experimental de Silvicultura Tropical, Instituto Nacional de Pesquisas da Amazônia, durante o período de outubro de 1998 a março de 1999.

\begin{tabular}{|c|c|c|c|c|c|c|c|c|}
\hline \multirow[t]{2}{*}{ Espécies } & \multicolumn{8}{|c|}{ Percentual de espécies coletados por altura } \\
\hline & $1 \mathrm{~m}$ & $\%$ & $10 \mathrm{~m}$ & $\%$ & $20 \mathrm{~m}$ & $\%$ & Total & $\%$ \\
\hline B. pintoi & 0 & 0,0 & 2 & 0,2 & 0 & 0,0 & 2 & 0,07 \\
\hline L. amazonensis & 13 & 1,5 & 71 & 6,9 & 5 & 0,5 & 89 & 3,11 \\
\hline L. anduzei & 60 & 7,1 & 68 & 6,6 & 365 & 37,1 & 493 & 17,2 \\
\hline L. aragaoi & 6 & 0,7 & 11 & 1,1 & 2 & 0,2 & 19 & 0,66 \\
\hline L. ayrozai & 12 & 1,4 & 77 & 7,5 & 24 & 2,4 & 113 & 3,95 \\
\hline L. bispinosa & 0 & 0,0 & 2 & 0,2 & 0 & 0,0 & 2 & 0,07 \\
\hline L. cayennensis & 1 & 0,2 & 0 & 0,0 & 0 & 0,0 & 1 & 0,03 \\
\hline L. choti & 10 & 1,2 & 6 & 0,6 & 0 & 0,0 & 16 & 0,56 \\
\hline L. davisi & 19 & 2,2 & 199 & 19,3 & 25 & 2,5 & 243 & 8,5 \\
\hline L. dendrophyla & 6 & 0,7 & 7 & 0,7 & 28 & 2,8 & 41 & 1,4 \\
\hline L. dreisbachi & 2 & 0,2 & 25 & 2,4 & 15 & 1,5 & 42 & 1,5 \\
\hline L. flaviscutellata & 20 & 2,4 & 6 & 0,6 & 3 & 0,3 & 29 & 1,01 \\
\hline L. furcata & 15 & 1,8 & 2 & 0,2 & 44 & 4,5 & 61 & 2,1 \\
\hline L. geniculata & 11 & 1,3 & 68 & 6,6 & 15 & 1,5 & 94 & 3,3 \\
\hline L. gomezi & 12 & 1,4 & 11 & 1,0 & 14 & 1,4 & 37 & 1,3 \\
\hline L. inflata & 0 & 0,0 & 1 & 0,1 & 0 & 0,0 & 1 & 0,03 \\
\hline L. infraspinosa & 59 & 7,0 & 12 & 1,2 & 1 & 0,1 & 72 & 2,5 \\
\hline L. inpai & 0 & 0,0 & 5 & 0,5 & 0 & 0,0 & 5 & 0,2 \\
\hline L. lutziana & 2 & 0,2 & 9 & 0,9 & 1 & 0,1 & 12 & 0,4 \\
\hline L. migonei & 3 & 0,3 & 12 & 1,2 & 10 & 1,0 & 25 & 0,87 \\
\hline L. monstruosa & 29 & 3,4 & 2 & 0,2 & 2 & 0,2 & 33 & 1,15 \\
\hline L. nematoducta & 28 & 3,3 & 6 & 0,6 & 3 & 0,3 & 37 & 1,3 \\
\hline L. olmeca nociva & 74 & 8,7 & 12 & 1,2 & 0 & 0,0 & 86 & 3,0 \\
\hline L. pacae & 2 & 0,2 & 0 & 0,0 & 0 & 0,0 & 2 & 0,07 \\
\hline L. paraensis & 8 & 0,9 & 33 & 3,2 & 8 & 0,8 & 49 & 1,7 \\
\hline L. pennyi & 3 & 0,3 & 0 & 0,0 & 0 & 0,0 & 3 & 0,1 \\
\hline L. pilosa & 0 & 0,0 & 1 & 0,1 & 1 & 0,1 & 2 & 0,07 \\
\hline L.raticliffei & 4 & 0,5 & 6 & 0,6 & 0 & 0,0 & 10 & 0,35 \\
\hline L rorotaensis & 135 & 16,0 & 52 & 5,0 & 20 & 2,0 & 207 & 7,24 \\
\hline L. ruii & 6 & 0,7 & 1 & 0,1 & 12 & 1,22 & 19 & 0,7 \\
\hline L. sericea & 30 & 3,5 & 16 & 1,6 & 5 & 0,5 & 51 & 1,8 \\
\hline L. shannoni & 3 & 0,3 & 1 & 0,1 & 1 & 0,1 & 5 & 0,2 \\
\hline L. squamiventris & 17 & 2,0 & 40 & 3,9 & 5 & 0,5 & 62 & 2,2 \\
\hline L. trichopyga & 62 & 7,4 & 54 & 5,2 & 6 & 0,6 & 122 & 4,3 \\
\hline L. trispinosa & 3 & 0,3 & 2 & 0,2 & 0 & 0,0 & 5 & 0,2 \\
\hline L. tuberculata & 7 & 0,8 & 0 & 0,0 & 43 & 4,4 & 50 & 1,7 \\
\hline L. umbratilis & 158 & 18,6 & 163 & 15,8 & 313 & 31,8 & 634 & 22,2 \\
\hline Grupo Verrucarum & 0 & 0,0 & 0 & 0,0 & 2 & 0,2 & 2 & 0,07 \\
\hline Lutzomyia sp. & 27 & 3,2 & 45 & 4,4 & 11 & 1,1 & 83 & 2,9 \\
\hline Total & 847 & 100,0 & 1.028 & 100,0 & 984 & 100,0 & 2.859 & 100,0 \\
\hline
\end{tabular}


Figura 4

Estratificação vertical das espécies do sub-gênero Nyssomyia, coletadas na BR 174, Estação Experimental

de Silvicultura Tropical, Instituto Nacional de Pesquisas da Amazônia, com armadilhas CDC, durante os meses de outubro de 1998 a março de 1999.

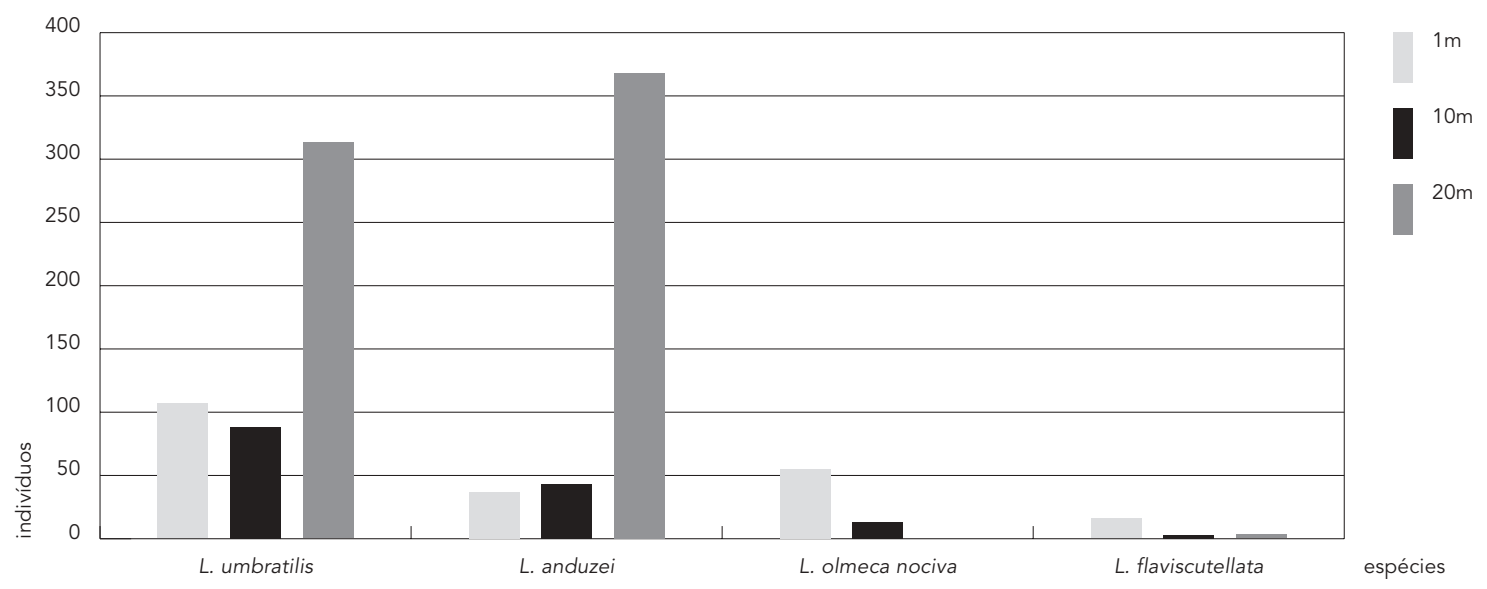

Figura 5

Estratificação vertical das espécies do sub-gênero Psychodopygus, capturadas com armadilhas CDC, na BR 174, Estação Experimental de Silvicultura Tropical, Instituto Nacional de Pesquisas da Amazônia, durante os meses de outubro de 1998 a março de 1999.

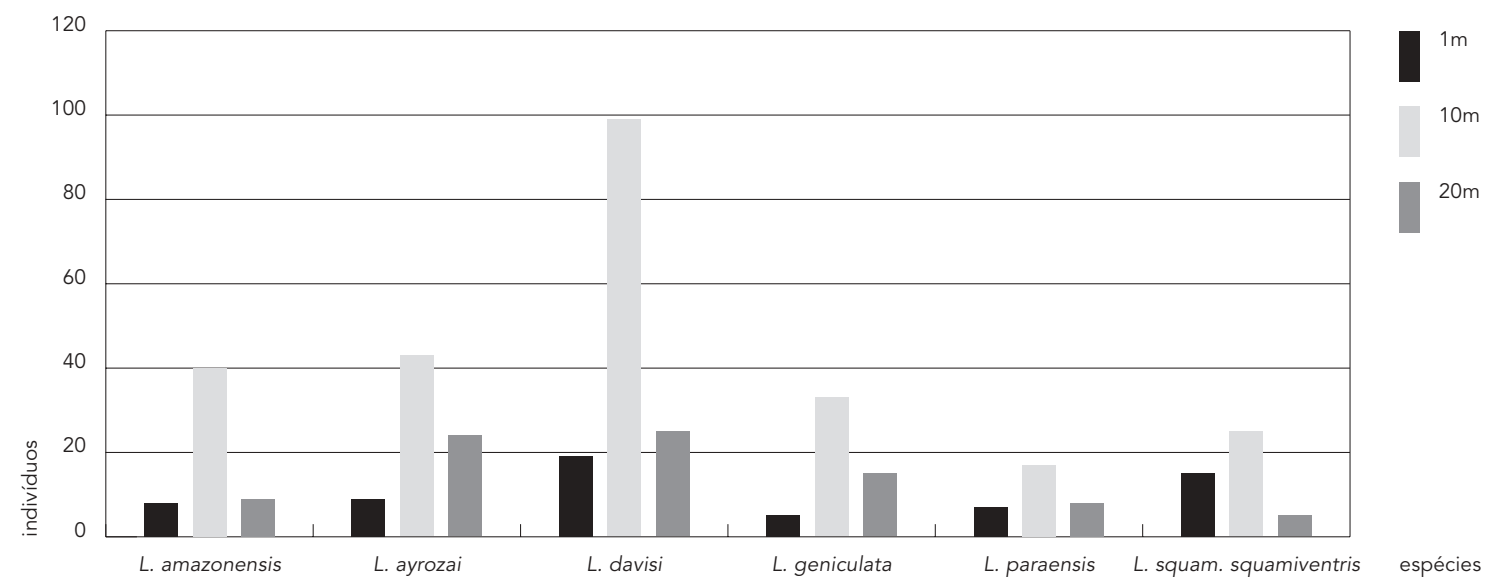


Figura 6

Estratificação vertical das espécies de flebótomos, de diferentes sub-gêneros, capturadas com armadilhas CDC, na BR 174, Estação Experimental de Silvicultura Tropical, Instituto Nacional de Pesquisas da Amazônia, durante os meses de outubro de 1998 a março de 1999.

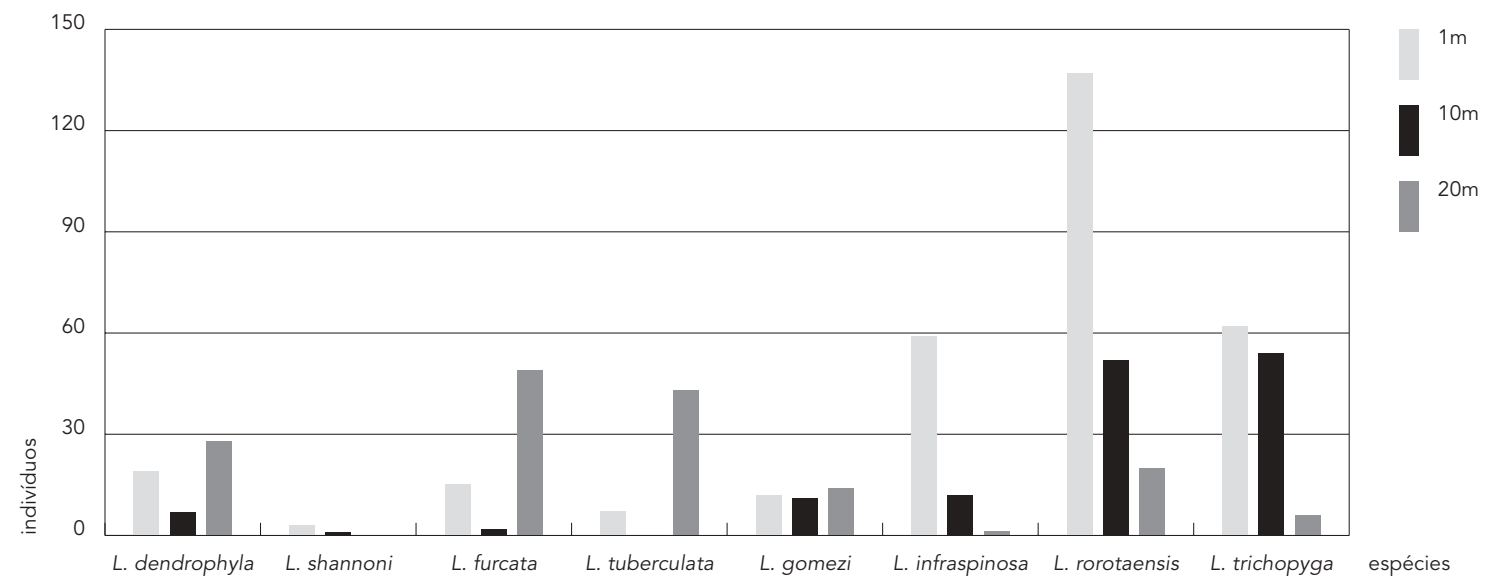

pouco representativas nas armadil has colocadas a $1 \mathrm{~m}$. Já as espécies L. rorotaensis, L. flaviscutellata, L. monstruosa, L. ol meca nociva, também concordando com resultados deste trabaIho, foram capturadas em maior número nas armadilhas colocadas a $1 \mathrm{~m}$ de altura.

Segundo Arias \& Freitas (1982), há evidências da preferência dos flebótomos da Amazônia Central pelas copas das árvores, em relação ao solo da floresta, quando observaram a presença de $86,6 \%$ dos flebótomos a $15 \mathrm{~m}$ de altura, onde predominaram L. umbratilise L. anduzei. Esse fato também foi observado por Ready et al. (1986) no Pará e Castellón et al. (1989) em Roraima, onde essas duas espécies também foram mais abundantes em grandes alturas, quando a floresta era alta com as copas interligadas, do que ao nível do solo.

Quanto à espécie L. squamiventris nas coletas de Arias \& Freitas (1982), foi mais freqüente nas armadilhas colocadas a $1 m$, enquanto que na nossa área de estudos comportou-se de forma diferente, sendo mais coletadas nas armadilhas colocadas a $10 \mathrm{~m}$, juntamente com as outras espécies do sub-gênero Psychodopygus, concordando com os resultados obtidos por Castellón et al. (1989).

Semel hante aos resultados obtidos por Barrett (1993), L. umbratilis foi dominante em todas as alturas. Segundo o mesmo autor, a concentração do sub-gênero Psychodopygus em baixas alturas, pode ajudar nas buscas dos sí- tios de repouso das fêmeas oníparas, facilitando as observações das relações parasito-vetor e vetor-hospedeiro.

Genaro et al. (1986), estudando a estratificação vertical dos flebótomos em Balbina, Amazonas, coletaram 45 espécies diferentes de flebótomos e demonstraram que a maioria delas ocorria abaixo de $10 \mathrm{~m}$ de altura. Neste trabalho, um maior número de indivíduos também ocorreu nas armadilhas colocadas até $10 \mathrm{~m}$ de altura.

Ready et al. (1983) citaram que as espécies L. infraspinosa e L. pacae Floch \& Abonnenc, 1943, são fortemente rodentofílicos, enquanto que L. choti , L. trichopyga e L. aragaoi são comuns em buracos de tatus. Os resultados mostraram relação com essas afirmações, pois a maioria dos exemplares dessas espécies foi capturada a $1 \mathrm{~m}$, decrescendo a freqüência a $10 \mathrm{~m}$, tornando-se raros ou ausentes a $20 \mathrm{~m}$.

As espécies do sub-gênero Psychodopygus, na sua maioria consideradas antropofílicas, L. paraensis, L. davisi, L. amazonensis, L. squamiventris squamiventris e $L$. ayrozai , foram coletadas com maior freqüência a $10 \mathrm{~m}$ de altura do solo. Esses resultados podem indicar que estas espécies estejam se dispersando ativamente à procura de vertebrados que habitam mais ao nível do solo, para realização do hematofagismo.

As espécies L. ayrozai e L. davisi recentemente foram envolvidas na transmissão de L. naiffi Lainson \& Shaw, 1989, uma leishmania 
isolada do tatu Dasypus novencictus, na região do Amazonas (Lainson \& Shaw, 1989).

Os flebótomos do sub-gênero Psathyromyia, L. dendrophyla, L. scaffi e L. shannoni, foram pouco atraídos por armadilhas de luz em Bal bina (Barrrett, 1993), o que confirmamos em nossas coletas. Essas espécies, principalmente L. shannoni, têm sido associadas com a transmissão de Endotrypanum schaudinni Mesnil \& Brimont 1908, um tripanosomatídeo exclusivo de preguiças (Arias et al., 1985).

Pelo teste de precipitina, Christensen et al. (1982), demonstraram a atração de L. umbratilis pelos edentados (64\%, principalmente por C. didactylus), L. shannoni, similar a espécie anterior com $73 \%$ para preguiças e L. anduzei por Bradypodidae (63\%). Tesh et al., (1971, 1972) no Panamá, realizaram também testes de precipitina em L. umbratilis e demonstraram que, de 39 exemplares examinados, 20 (51,3\%) tinham se alimentado em primatas, 11 (28\%) em roedores e 8 (18\%) em tamanduás. Noutra ocasião, contudo, verificaram que 35 exemplares de L. umbratilis tinham se alimentado sobre primatas e um sobre porco-espinho (Lainson \& Shaw, 1979).

A espécie L. rorotaensis, tem sido encontrada no Estado do Pará, ocasionalmente infectada com Trypanosoma tetradactyli, provavelmente de répteis da espécie Thecadactylus rapicaudatus (Lainson \& Shaw, 1979). Estudos usando técnicas moleculares (KDNA) para a detecção de parasitos em flebotomíneos, também demonstraram infecção por Endotrypanum em L. shannoni, L. umbratilise L. anduze (Franco \& Grimaldi, 1999).

No Pará, L. tuberculata foi encontrada com uma espécie desconhecida de leishmania, assinalada no sub-gênero Viannia (Killick-Kendrick, 1990).

Ainda, segundo este autor, 16 isolados de L. umbratis, 1 de L. ayrozi e 13 de L. squamiventris squamiventristambém não foram identificados.

A presença mais freqüente das espécies $L$. flaviscutellata e $L$ olmeca nociva, vetoras da Leishmania (Leishmania) amazonensis Lainson \& Shaw, 1972, ao nível do solo, tem uma relação direta com sua associação por roedores silvestres, Proechimys e Oryzomys, encontrados naturalmente infectados com a leishmania citada. Por sua vez, L. umbratilis e L. anduzei, vetoras da Leishmania (Viannia) guyanensis Floch, 1954, e que são atraídas por Edentados Chol oepus di dactylus e marsupiais do gênero Didel phis, animais arborícolas, que sobem e descem as árvores em busca de alimento, foram encontradas com freqüência nos três níveis de estratificação.

Rangel et al. (1999) estudando a distribuição vertical de flebótomos, demonstraram que, tanto ao nível do solo quanto na copa das árvores, a espécie L. umbratilis, juntamente com L. whitmani el. antunesi eram dominantes.

Esses resultados, assim como os obtidos por outros autores, demonstram que ao longo da estratificação vertical, a distribuição dos flebótomos variou em número de espécies, o que pode ser explicada em parte, por exemplo, pela preferência alimentar de espécies como L. umbratilis por preguiças ao nível da copa das árvores e L. flaviscutellata e L. ol meca nociva por roedores, ao nível do solo (Genaro et al., 1986).

A espécie L. umbratilis, principal vetor de L. guyanensis no norte do Brasil e nas Guianas, tem os troncos de árvores como seus lugares habituais de descanso, e as fêmeas se movem periodicamente desde o nível do solo até as copas das árvores, onde ficam hospedeiros reservatórios como Choloepus didactyluse Tamanduá tetradactyla (Lainson, 1983; Young \& Arias, 1992). Quando os flebótomos, como por exemplo L. umbratilis, são perturbados nos troncos, tanto no nível do solo como na copa das árvores, podem vir a transmitir para o homem a $\mathrm{L}$. guyanensis (Bal bino et al., 2001).

Os resultados obtidos em relação à espécie L. umbratilis, sendo encontrada em quantidades si gnificativas nos diferentes níveis de estratificação vertical, desde o solo até as copas das árvores, são importantes para a compreensão da epidemiologia da propagação das doenças transmitidas pelos flebótomos para o homem. O risco da transmissão de L. guyanensis por L. umbratilis pode ocorrer durante os períodos diurno e noturno, quando o vetor é perturbado em seus sítios de repouso.

\section{Agradecimentos}

Ao Professor Rui Alves de Freitas (Coordenação de Pesquisas em Ciências da Saúde, Instituto Nacional de Pesquisas da Amazônia), pelo auxílio na identificação específica dos flebótomos. 


\section{Referências}

AGUIAR, G. M.; VILELA, M. L.; SCHUBACK, P.; SOUCASAUX, T. \& AZEVEDO, A. C., 1985. Aspectos da ecologia dos flebótomos do Parque Nacional da Serra dos Órgãos, Rio de Janeiro. IV. Freqüência mensal em armadilhas luminosas (Diptera: Psychodidae: Phlebotominae). Memórias do Instituto Oswal do Cruz, 80:465-482.

ARIAS, J. R. \& FREITAS, R. A., 1982. On the vectors of cutaneous leishmaniasis in the Central Amazon of Brazil. 3. Phlebotomine sand fly stratification in a terra firme forest. Acta Amazonica, 12:599-603.

ARIAS, J. R.; MILES, M. A.; NAIFFI, R. D.; POPOVA, M. M.; FREITAS, R. A.; BIANCARDI, C. B. \& CASTELLON, E. G., 1985. Flagellates infections of Brazilian sandflies (Diptera: Psychodidae): Isolation in vitro and biochemical identification of Endotrypanum e Leishmania. American Journal of Tropical Medicineand Hygiene, 34:1098-1108.

BALBINO, V. Q.; MARCONDES, C. B.; ALEXANDER, B.; LUNA, L. X. S.; LUCENA, M. M. M.; MENDES, A. C. S. \& ANDRADE, P. P., 2001. First report of Lutzomyia (Nyssomyia) umbratilis Ward \& Frahia, 1977 outside of Amazonian region, in Recife, State of Pernambuco, Brazil. (Diptera: Psychodidae: Phlebotominae). Memórias do Instituto Oswaldo Cruz, 96:315-317.

BARRETT, T. V., 1993. Cutaneous leishmaniasis in Amazonas State, Brazil: Eco-epidemiology and questions of control. In: Research and Control of Leishmaniosis in Brazil: Proceedings of National Workshop (S. P. Brandão Filho, ed.), pp. 31-44, Recife: Centro de Pesquisas Aggeu Magalhães, Fundação Oswaldo Cruz.

BIANCARDI, C. B., 1981. Aspectos da Epidemiologia da Leishmaniose Cutânea na Rodovia BR 364, Território Federal de Rondônia. Dissertação de Mestrado, Manaus: Instituto Nacional de Pesquisas da Amazônia/Universidade do Amazonas.

CABANILLAS, M. R. S. \& CASTELLON, E. G., 1999. Distribution of sandflies (Diptera: Psychodidae) on tree-trunks in a non-flooded area of the Ducke Forest Reserve, Manaus, AM, Brazil. Memórias do Instituto Oswaldo Cruz, 94:289-296.

CASTELLON, E. G.; ARAUJO FILHO, N. A.; FÉ, N. F. \& ALVES, J. M. C., 1989. Flebótomos (Díptera: Psychodidae) no Estado de Roraima, Brasil. I. Espécies coletadas nas regiões Sul e Central. Memórias do Instituto Oswaldo Cruz, 84:95-99.

CASTELLON, E. G.; ARAUJO FILHO, N. A.; FÉ, N. F. \& ALVES, J. M. C., 1991. Flebótomos (Díptera: Psychodidae) no Estado de Roraima, Brasil. II. Espécies coletadas na região Norte. Acta Amazonica, 21:45-50.

CASTELLON, E. G.; ARIAS, J. R.; FREITAS, R. A. \& NAIFF, R. D., 1994. Os flebótomos da região Amazônica, estrada Manaus-Humaitá, estado do Amazonas, Brasil (Diptera; Psychodidae; Phlebotominae). Acta Amazonica 24:91-102.

CHANIOTIS, B. N.; CORREA, M. A.; TESH, R. B. \& JONHSON, K. M., 1971a. Daily and seasonal manbiting activity of Phlebotomine sandflies in Panama. Journal of Medical Entomology, 8:415-420.

CHANIOTIS, B. N.; NEELY, J. M.; CORREA, M. A.; TESH, R. B. \& JONHSON, K. M., 1971b. Natural population dynamics of Phlebotomine sandflies in Panama. Journal of Medical Entomology, 8:339352.

CHRISTENSEN, H. A.; ARIAS, J. R.;VASQUEZ, A. M. \& FREITAS, R. A., 1982. Host of sandfly vectors of Leishmania braziliensis guyanensis in the Central Amazon of Brazil. American Journal of Tropical Medicine and Hygiene, 31:239-242.

DISNEY, R. H. L., 1966. A trap for phlebotomine sandflies attracted to rats. Bulletin of Entomology Research, 56:445-451.

FRANCO, A. M. R. \& GRIMALDI, G., 1999. Characterization of Endotrypanum (Kinetoplastida: Trypanosomatidae), a unique parasite infecting the neotropical tree sloths (Edentata). Memórias do Instituto Oswaldo Cruz, 94:261-268.

GENARO, O.; FREITAS, R. A.; NAIFF, R. D. \& ARIAS, J. R., 1986. Estratificação vertical de vetores da leishmaniose em floresta de terra firme, Amazonas. Revista da Sociedade Brasileira de Medicina Tropical, 19(Sup. 1):79.

GEOFFROY, B.; DEDET, J. P.; LEBBE, J.; ESTERRE, P. \& TRAPE, J. F., 1986. Note sur les relations des vecteurs de leishmaniose avec les essences forestieres en Guyane Française. Annales de Parasitologie Humaine et Comparée, 61:491-505.

JARDIM, F. C. S. \& HOSAKAWA, R. T., 1987. Estrutura da floresta equatorial úmida da Estação de Silvicultura Tropical do INPA. Acta Amazonica, 16/17: 411-508.

KILLICK-KENDRICK, R., 1990. Phlebotomine vectors of the leishmaniases: A rewiew. Medical and Veterinary Entomology, 4:1-24.

LAINSON, R., 1983. The American Leishmaniasis: Some observation on their ecology and epidemiology. Transactions of the Royal Society of Tropical Medicineand Hygiene, 77:569-596.

LAINSON, R. \& SHAW, J. J., 1979. The role of animals in the epoidemiology of South American leishmaniasis. In: Biology of the Kinetoplastida (W. H. R. Lumsdem \& D. A. Evans, ed.), v. 2, pp. 1-116, London/New York: Academic Press.

LAINSON, R. \& SHAW, J. J., 1989. Leishmania (viannia) naiffi sp.n. A parasite of the armadillo, Dasypusnovencinctus (L.) in Amazonian Brazil. Annales de Parasitologie Humaine et Comparée, 64:3-9.

RANGEL, E. F.; AZEVEDO, A. C. R.; LIMA, J. B.; SOUZA, N. A.; PEREIRA, T.; MANESES, C. R. V. \& COSTA, W. A., 1999. Ecologia da leishmaniose cutânea no Estado de Mato Grosso. I. Distribuição vertical da fauna flebotomínica (Diptera: Psychodidae: Phlebotominae). Revista da Sociedade Brasileira de Medicina Tropical, 32(Sup. 1):25-26.

READY, P. D.; LAINSON, R. \& SHAW, J. J ., 1983. Leishmaniasis in Brazil: XX. Prevalence of enzootic rodent leishmaniasis (Leishmania mexicana amazonensis) and apparent absence of "pian bois" (Leishmania braziliensis guyanensis) in plantations of introduced tree species and others nonclimax forests in eastern Amazônia. Transactions of the Royal Soci ety of Tropical Medicineand Hygiene, 77:775-785.

READY, P. D.; LAINSON, R.; SHAW, J. J. \& WARD, R. D., 1986. The ecology of Lutzomyia umbratilisWard 
\& Fraiha (Diptera: Psychodidae) the major vector to man of Leishmania braziliensis guyanensis in north-eastern Amazonian Brazil. Bulletin of Entomological Research, 76:21-40.

RUTLEDGE, L. C. \& ELLENWOOD, D. A., 1975. Production of phlebotomine sandflies on the open forest floor in Panama: Phytologic and Edaphic relations. Environmental Entomology, 4:83-89.

SHAW, J. J.; LAINSON, R. \& WARD, R. D., 1972. Leishmaniasis in Brazil. VII. Further observations on the feeding habits of Lutzomyia flaviscutellata (Mangabeira) with particular reference to its biting habits at different heights. Transactions of the Royal Society of Tropical Medicine and Hygiene, 66:718-723.

SHERLOCK, I. A.; M AIA, H. \& DIAS-LIMA, A. G., 1996. Resultados preliminares de um projeto sobre a ecologia dos flebotomíneos vetores de leishmaniose tegumentar no Estado da Bahia. Revista da Sociedade Brasileira de Medicina Tropical, 29: 207-214.

SILVA, B. M., 1993. Levantamento da Fauna Flebotômica da Reserva Florestal Ducke usando Diferentes Tipos de Armadil has e Iscas. Dissertação de Mestrado, Manaus: Instituto Nacional de Pesquisas da Amazônia/Universidade do Amazonas.

TESH, R. B.; CHANIOTIS, B. N.; ARONSON, M. B. \& JOHNSON, K. M., 1971. Natural host preferences of Panamanian phlebotomine sandflies as determined by precipitin test. American Journal of Tropical Medicineand Hygiene, 20:150-156.
TESH, R. B.; CHANIOTIS, B. N.; ARONSON, M. B. \& JOHNSON, K. M., 1972. Further studies on the natural host preferences of Panamanian phlebotomine sandflies. American Journal of Epidemiology, 95:88-93.

WILLIAMS, P., 1970. Phlebotomine sand flies and leishmaniasis in British Honduras (Belize). Transactions of the Royal Society of Tropical Medicine and Hygiene, 64:317-364.

YABE, T.; RITTL, C. E. \& HIGICHI, N., 1998. Espécies de mamíferos registrados por câmeras fotográficas na Estação Experimental de Silvicultura Tropical do INPA - EEST - INPA, Amazônia Central. In: Pesquisas Florestais para a Conservação da Floresta e Reabilitação das Áreas Degradadas da Amazônia (N. Higuchi, M. Campos, P. T. B. Sampaio \&J. Santos, org.), pp. 95-107, Manaus: Instituto Nacional de Pesquisas da Amazônia.

YOUNG, D. G. \& ARIAS, J. R., 1992. Flebótomos Vectores de Lei shmaniasis en las Américas. Caderno Técnico 33. Washington, DC: Organización Panamericana de Salud.

Recebido em 25 de maio de 2001

Versão final reapresentada em 8 de novembro de 2001 Aprovado em 22 de janeiro de 2002 